\title{
Lithium Chloride Reinforces the Regeneration-Promoting Effect of Chondroitinase ABC on Rubrospinal Neurons after Spinal Cord Injury
}

\author{
LEUNG-WAH YICK, ${ }^{1,2}$ KWOK-FAI SO, ${ }^{1}$ PIK-TO CHEUNG, ${ }^{2}$ and WU-TIAN WU ${ }^{1}$
}

\begin{abstract}
After spinal cord injury, enzymatic digestion of chondroitin sulfate proteoglycans promotes axonal regeneration of central nervous system neurons across the lesion scar. We examined whether chondroitinase $\mathrm{ABC}(\mathrm{ChABC})$ promotes the axonal regeneration of rubrospinal tract (RST) neurons following injury to the spinal cord. The effect of a GSK-3 $\beta$ inhibitor, lithium chloride (LiCl), on the regeneration of axotomized RST neurons was also assessed. Adult rats received a unilateral hemisection at the seventh cervical spinal cord segment (C7). Four weeks after different treatments, regeneration of RST axons across the lesion scar was examined by injection of Fluoro-Gold at spinal segment T2, and locomotor recovery was studied by a test of forelimb usage. Injured RST axons did not regenerate spontaneously after spinal cord injury, and intraperitoneal injection of $\mathrm{LiCl}$ alone did not promote the regeneration of RST axons. Administration of ChABC at the lesion site enhanced the regeneration of $\mathrm{RST}$ axons by $20 \%$. Combined treatment of $\mathrm{LiCl}$ together with $\mathrm{ChABC}$ significantly increased the regeneration of RST axons to $42 \%$. Animals receiving combined treatment used both forelimbs together more often than animals that received sham or single treatment. Immunoblotting and immunohistochemical analysis revealed that $\mathrm{LiCl}$ induced the expression of inactive GSK-3 $\beta$ as well as the upregulation of Bcl-2 in injured RST neurons. These results indicate that in vivo, $\mathrm{LiCl}$ inhibits $\mathrm{GSK}-3 \beta$ and reinforces the regeneration-promoting function of $\mathrm{ChABC}$ through a Bcl-2-dependent mechanism. Combined use of $\mathrm{LiCl}$ together with $\mathrm{ChABC}$ could be a novel treatment for spinal cord injury.
\end{abstract}

Key words: axonal regeneration; behavioral analysis; chondroitin sulfate; lithium; rubrospinal tract; spinal cord injury

\section{INTRODUCTION}

$\mathbf{N}$ EURONS IN THE ADULT mammalian spinal cord fail to regenerate axons after injury, resulting in permanent functional deficits. Modulation of the extrinsic environment and modeling of the intrinsic response are both em- ployed as strategies for promoting regeneration and recovery of injured central nervous system (CNS) axons. Specific examples include grafts of peripheral nerves (Richardson et al., 1982), fetal CNS tissue (Himes et al., 1994; Diener and Bregman, 1998), or genetically modified nonneuronal cells (Jin et al., 2002); neutralization of

Departments of ${ }^{1}$ Anatomy and ${ }^{2}$ Paediatrics and Adolescent Medicine, Faculty of Medicine, The University of Hong Kong, Hong Kong. 


\section{LITHIUM AND CHONDROITINASE ABC PROMOTE REGENERATION}

growth-inhibitory molecules (Caroni and Schwab, 1988; Schnell and Schwab, 1990; Thallmair et al., 1998); application of neurotrophic factors (Kobayashi et al., 1997; Shibayama et al., 1998); and overexpression of growthpromoting genes (Mason et al., 2002).

Injury to the spinal cord elicits robust glial reactions and subsequent formation of gliotic scar (Schwab et al., 2001; Liesi and Kauppila, 2002). Chondroitin sulfate proteoglycans (CSPGs) are one of the inhibitory components of the gliotic scar that hinder the regeneration of severed axons (Dou and Levine, 1994; Bovolenta et al., 1997; Fidler et al., 1999). Following spinal cord injury, upregulation of CSPGs has been found in the gliotic scar surrounding the lesion site (Wang et al., 1997). We and others have recently demonstrated that in vivo, enzymatic degradation of CSPGs at the lesion site can significantly promote the regeneration of severed axons across the gliotic scar in injured brain and spinal cord (Yick et al., 2000, 2003; Moon et al., 2001; Bradbury et al., 2002).

Lithium has been used for the treatment and prophylaxis of mania and depression in humans for almost half a century. Increasing numbers of studies have indicated that lithium has neuroprotective effects (Nonaka et al., 1998; Chen and Chuang, 1999; Wei et al., 2001). However, the molecular mechanisms underlying the actions of lithium have not been fully elucidated. Recent studies have shown that lithium exerts effects via a number of intracellular signaling pathways, including upregulation of Bcl-2 and direct inhibition of glycogen synthase kinase-3 $\beta$ (GSK-3 $\beta$ ) (Chen et al., 1999; Manji et al., 2000). It has been suggested that lithium-induced Bcl-2 upregulation plays a crucial role in neuroprotection against glutamate excitotoxicity (Chen and Chuang, 1999). Bcl-2 also supports the intrinsic growth potential of injured axons (Chierzi et al., 1999; Holm and Isacson, 1999). GSK$3 \beta$ is a key downstream target of the PI3-kinase/Akt signaling pathway that regulates apoptosis (Pap and Cooper, 1998; Crowder and Freeman, 2000). Neuronal apoptosis induced by trophic factor withdrawal is associated with an increase in GSK-3 $\beta$ activity (Hetman et al., 2000).

A severed rubrospinal tract (RST) can regenerate its axons under appropriate conditions (e.g. following application of neurotrophic factors; Xu and Martin, 1991; Liu et al., 1999; Jin et al., 2002). The RST is a descending pathway that has an almost complete contralateral trajectory, allowing unambiguous interpretations of the axonal tracing results (Brown, 1974). In this study, we show that enzymatic degradation of CSPGs by chondroitinase $\mathrm{ABC}(\mathrm{ChABC})$ can promote the axonal regeneration of RST after spinal cord injury. Interestingly, we demonstrate for the first time that the use of lithium chloride (LiCl), a GSK-3 $\beta$ inhibitor, can markedly reinforce the regeneration-promoting effect of $\mathrm{ChABC}$ on axotomized
RST neurons, resulting in a significant recovery of forelimb function.

\section{MATERIALS AND METHODS}

\section{Surgical Procedures}

Adult 9-week-old Sprague-Dawley rats (200-250 g) were anesthetized with intraperitoneal injection of ketamine $(80 \mathrm{mg} / \mathrm{kg})$ and xylazine $(8 \mathrm{mg} / \mathrm{kg})$. Under an operating microscope, a dorsal laminectomy was carried out and the seventh cervical spinal cord segment (C7) was identified. After opening the dura mater, a right hemisection was performed at this level, using a pair of spring scissors.

Following spinal cord hemisection, animals were divided into four groups. In the first group, animals received a piece of gelfoam soaked with $0.1 \mathrm{M}$ phosphate-buffered saline (PBS), placed on top of the lesion site, together with a daily intraperitoneal injection of $1.0 \mathrm{~mL}$ PBS to serve as the sham control. In the second group, animals received a gelfoam soaked with PBS, placed on top of the lesion site, together with daily intraperitoneal injections of 1.0 $\mathrm{mL}$ of $85 \mathrm{mg} / \mathrm{kg} \mathrm{LiCl}$. In the third group of animals, a small piece of gelfoam soaked with $10 \mu \mathrm{L}$ of 2.5 units $/ \mathrm{mL}$ of Proteus vulgaris ChABC lyase (Seikagaku Corporation) was placed on top of the lesion site. The animals also received daily intraperitoneal injections of $1.0 \mathrm{~mL}$ PBS. In the fourth group, animals received a combined treatment of daily intraperitoneal injections of $85 \mathrm{mg} / \mathrm{kg}$ $\mathrm{LiCl}$ together with a piece of gelfoam soaked with $10 \mu \mathrm{L}$ of 2.5 units $/ \mathrm{mL}$ of $\mathrm{ChABC}$ placed on top of the lesion site. We have shown that intraperitoneal injection of 85 $\mathrm{mg} / \mathrm{kg} \mathrm{LiCl}$ can protect retinal ganglion cells in a rat glaucoma model (unpublished observations). In addition, $\mathrm{ChABC}$ at 2.5 units/mL enhances the axonal regrowth of Clarke's neurons after spinal cord injury (Yick et al., 2000, 2003). After operation, animals were allowed to survive for 1 month and were then further subdivided into subgroups for either axonal tracing $(n=5)$ or behavioral analysis $(n=4)$. Two additional groups of animals received daily intraperitoneal injection of either PBS or 85 $\mathrm{mg} / \mathrm{kg} \mathrm{LiCl}$ and were used for immunohistochemistry and Western blot analysis. Animals for immunohistochemistry and Western blot were allowed to survive for either 1 or 5 days ( $n=8$ for each time point, $n=4$ for immunohistochemistry, and $n=4$ for Western blot).

\section{Retrograde Labeling of Regenerated RST Neurons}

Fluoro-Gold (FG, 6\% w/v, Fluorochrome) was used to label the RST neurons that had regenerated their axons 


\section{YICK ET AL.}

across the injury scar and that reentered the caudal spinal cord. Two days before the end of the postinjury survival period (1 month), animals were anesthetized with intraperitoneal injection of ketamine $(80 \mathrm{mg} / \mathrm{kg})$ and $\mathrm{xy}-$ lazine $(8 \mathrm{mg} / \mathrm{kg})$. A dorsal laminectomy was then carried out, and a second thoracic (T2) spinal segment was identified. FG at a volume of $0.5 \mu \mathrm{L}$ was manually injected into the right $\mathrm{T} 2$ spinal cord using a Hamilton syringe. Because of the diffusibility of FG in the vicinity of the injection site and because a relatively large amount of FG $(0.5 \mu \mathrm{L})$ was used, this procedure actually labeled RST neurons in both sides.

The RST is a descending pathway in the superficial dorsolateral white matter of the spinal cord that has almost complete $(>99 \%)$ contralateral axonal projection without spared axons and collateral sprouting (Brown, 1974). Right hemisection at the C7 spinal cord severed axons from the left red nucleus. Except for rare ipsilateral projections ( $<1 \%$ of total RST neurons), injured RST neurons in the left midbrain with axons regenerating beyond the hemisection site to T2 were labeled by FG. Following C7 spinal cord hemisection, the ipsilateral intact side of the midbrain was used as an internal control.

\section{Tissue Preparation}

After the postinjury survival periods, animals were anesthetized with a lethal dose of ketamine and xylazine and were perfused intracardially with normal saline, followed by $400 \mathrm{~mL}$ of fixative containing $4 \%$ paraformaldehyde in 0.1 M PBS. The brain and spinal cord were removed, postfixed with paraformaldehyde overnight, and then placed in $30 \%$ phosphate-buffered sucrose. Brain and spinal cord tissues were cut into $30-\mu \mathrm{m}$ sections on a cryostat and mounted onto gelatin-coated slides.

\section{Western Blot and Immunohistochemistry}

The effect of $\mathrm{LiCl}$ on the phosphorylation state of GSK-3 $\beta$ and the expression of Bcl-2 in RST neurons was examined both 1 and 5 days after the surgical operation. For Western blotting, the primary antibodies used were the polyclonal anti-p-GSK-3 $\beta$-serine-9 (Cell Signaling Technology, Beverly, MA), the monoclonal anti-GSK$3 \beta$ (Transduction Laboratories, Lexington, KY), and the monoclonal anti-Bcl-2 (Santa Cruz Biotechnology, Santa Cruz, CA). The injured and the contralateral intact side of the red nucleus were rapidly dissected and freshly frozen at the end of the postoperative survival periods. Samples of four rats per group were pooled, and the tissue was immediately placed in ice-cold lysis buffer (50 $\mathrm{mM}$ Tris-HCl, $150 \mathrm{mM} \mathrm{NaCl}, 4$ mM EDTA, pH 7.4) containing protease inhibitor cocktails (Sigma, St. Louis, MO), and was homogenized in a Vibra-Cell (Sonic and
Materials, Newtown, CT) homogenizer. The homogenate was centrifuged at $14,000 \times g$ for $40 \mathrm{~min}$ at $4^{\circ} \mathrm{C}$. The protein content was assayed by a Coomassie Brilliant Blue Protein Assay Reagent (Bio-Rad Laboratories, Richmond, CA). Total protein of $50 \mu \mathrm{g}$ was loaded onto $12.5 \%$ polyacrylamide gel with $3.0 \%$ stacking gel for electrophoresis. Proteins were transferred to nitrocellulose membrane (Protran, Schleicher \& Schuell, Keene, $\mathrm{NH})$ at $4^{\circ} \mathrm{C}$ overnight. The blots were rinsed in Trisbuffered saline (TBS, pH 7.5) containing 0.1\% Tween 20 (TBST) and were then blocked in TBST containing 5\% nonfat milk powder and $2 \%$ bovine serum albumin. These blots were then incubated with the primary antibody for $1 \mathrm{~h}$. The signals were visualized by species-specific horseradish peroxidase-conjugated secondary antibodies (Santa Cruz Biotechnology) and a chemiluminescent substrate (Amersham Biosciences, Arlington Heights, IL).

For immunohistochemistry, the primary antibodies used were the polyclonal anti-p-GSK- $3 \beta$-serine- 9 and the monoclonal anti-Bcl-2. Perfusion-fixed serial cross sections from the midbrain were preblocked with $2 \%$ normal serum with $0.1 \%$ Triton X-100 (Sigma) and then incubated at $4^{\circ} \mathrm{C}$ for $48 \mathrm{~h}$ with either anti-p-GSK-3 $\beta$ Ser9 $(1: 100)$ or anti-Bcl-2 (1:200) diluted in Tris-buffered saline (TBS, $\mathrm{pH} 7.4$ ) containing $2 \%$ normal serum with $0.1 \%$ Triton $\mathrm{X}-100$. The signal of the primary antibody was detected by TRITC-conjugated goat anti-rabbit-IgG (1:200 diluted in TBS) at room temperature for $1 \mathrm{~h}$. After rinsing in TBS, sections were coverslipped in fluorescent mounting medium (Dako, Carpenteria, CA). Control sections that did not receive the primary antibody were used to distinguish between specific and nonspecific staining.

\section{Image Analysis, Cell Quantification, and Statistics}

All images were captured using a SPOT color chargecoupled device camera (Diagnostic Instruments, Durham, NC) attached to a Leica fluorescent microscope. For the quantification of regenerated RST neurons, the number of FG-labeled RST neurons was counted in every other section throughout the rostrocaudal extent of the red nucleus (Kobayashi et al., 1997; Liu et al., 1999). Only the neurons with visible nuclei were counted, to avoid overestimation of the number of FG-labeled RST neurons. Counting was performed blind to the treatment of the animals. The number of FG-labeled RST neurons on the lesion side was expressed as a percentage of the total number of FG-labeled neurons on the contralateral intact side. For the survival of RST neurons after treatments, sections were counterstained with neutral red. Only the cells that were large in size and that possessed a stellate shape were counted as surviving neurons. Data were compared 


\section{LITHIUM AND CHONDROITINASE ABC PROMOTE REGENERATION}

among groups using one-way ANOVA followed by a Tukey-Kramer multiple comparisons test.

\section{Behavioral Analysis}

The use of forelimbs during spontaneous vertical exploration was examined 1 month after different treatments as described (Liu et al., 1999), with minor modifications. Rats were placed for $5 \mathrm{~min}$ in a clear Plexiglas cylinder (15 cm in diameter and $30 \mathrm{~cm}$ high) that encourages use of the forelimbs for vertical exploration. The following behaviors were scored: independent use of the left (unimpaired) or right (impaired) forelimbs for contacting the wall of the cylinder, and simultaneous use of both forelimbs to contact the wall of the cylinder. The assessments were made by two researchers blinded to the treatments. The vertical exploration behavior was expressed in terms of percentage use of unimpaired forelimb relative to the total number of impaired, unimpaired, and both limb use; percentage use of impaired forelimb relative to the total number of impaired, unimpaired, and both limb use; and percentage use of both forelimbs relative to the total number of impaired, unimpaired, and both limb use. The differences between groups were tested by one-way ANOVA followed by Bonferroni post hoc analysis.

\section{RESULTS}

\section{Regeneration of RST after ChABC Treatment}

One month after spinal cord hemisection, the regeneration of RST axons was studied using the FG retrograde tracing technique. On the lesioned side of red nucleus, almost no FG-labeled RST neurons were found in the sham control animals $(2.3 \pm 0.9 \%) 1$ month after the operation (Figs. 1 and 2). The few scattered FG-labeled RST neurons on the lesion side represented those that had ipsilateral axonal projections without being axotomized. After local application of 2.5 units/mL ChABC on top of the spinal cord hemisection site, there were $22.1 \pm 4.6 \%$ of the RST neurons on the lesion side labeled by FG. We did not found any observable differences on the number of large, stellate-shaped RST neurons on the injured side between the PBS- and ChABC-treated groups (data not shown). This result indicates that enzymatic degradation of CSPGs can promote the axonal regeneration of RST neurons beyond the lesion site after spinal cord injury.

\section{Effect of LiCl and ChABC on RST Regeneration}

After hemisection of the spinal cord, daily intraperitoneal injection of $85 \mu \mathrm{g} / \mathrm{kg} \mathrm{LiCl}$ for 1 month did not alter the percentage of FG-labeled RST neurons as compared with the sham control $(p>0.05)$ (Figs. 1 and 2).
However, combined treatment of application of ChABC at the lesion site together with daily injection of $\mathrm{LiCl}$ significantly increased the percentage of FG-labeled RST neurons to $41.5 \pm 6.0 \%(p<0.001$ compared with all the other treatment groups). No observable differences were found in the number of large, stellate-shaped RST neurons on the injured side between different treatment groups (data not shown).

To examine whether there were FG-labeled axons in the spinal cord lesion scar, horizontal sections from C7 were cut 1 month after spinal cord hemisection. In all groups, the spinal cord lesion cavity was filled with scar tissue without cystic spaces. There were no FG-labeled axons within the lesion site in either the sham or the $\mathrm{LiCl}$ treated animals (Fig. 3A,B). In animals treated with ChABC alone or with $\mathrm{LiCl}$ together with $\mathrm{ChABC}$, FGlabeled axons approximately 100-200 $\mu \mathrm{m}$ in length were found in the lesion scar (Fig. 3C,D). The morphology of the axons within the scar was similar in these two treatment groups.

\section{Phosphorylation of GSK-3 $\beta$ after LiCl Treatment}

Following spinal cord injury, Western blot analysis on lysates of the lesioned red nucleus revealed that the level of serine-9-phosphorylated GSK-3 $\beta$ increased 1 and 5 days after $\mathrm{LiCl}$ treatment (Fig. 4). The immunoreactivity of p-GSK- $3 \beta$-serine- 9 was barely detectable in the sham control. The signal of the phosphorylation-independent antibody, anti-GSK- $3 \beta$, remained stable in all groups. These results indicate that $\mathrm{LiCl}$ leads to the inactivation of GSK- $3 \beta$ via phosphorylation.

To localize the expression of inactive GSK- $3 \beta$, tissue sections from the midbrain were immunostained with the p-GSK-3 $\beta$-serine- 9 antibody. Inconsistent with the Western blotting result, intense immunoreactivity of the pGSK- $3 \beta$-serine- 9 was found on the injured side of the red nucleus 1 and 5 days after $\mathrm{LiCl}$ treatment (Fig. 5). The p-GSK- $3 \beta$-serine- 9 immunoreactivity was mainly observed in the cytoplasm of the RST neurons. There were no p-GSK- $3 \beta$-serine-9 immunopositive neurons found in the intact contralateral side of the red nucleus in LiCl-treated animals, nor in the injured side of the red nucleus of the sham control animals. These results indicate that daily intraperitoneal injection of $\mathrm{LiCl}$ inactivates GSK- $3 \beta$ by serine- 9 phosphorylation in the lesioned RST neurons after spinal cord injury.

\section{Expression of Bcl-2 after LiCl Treatment}

It has been reported that $\mathrm{Bcl}-2$ protects neurons from apoptotic cell death as well as enhances axonal regeneration after CNS injury (Chen et al., 1997; Yukawa et al., 2002; Huang et al., 2003). Here we have sought to ex- 


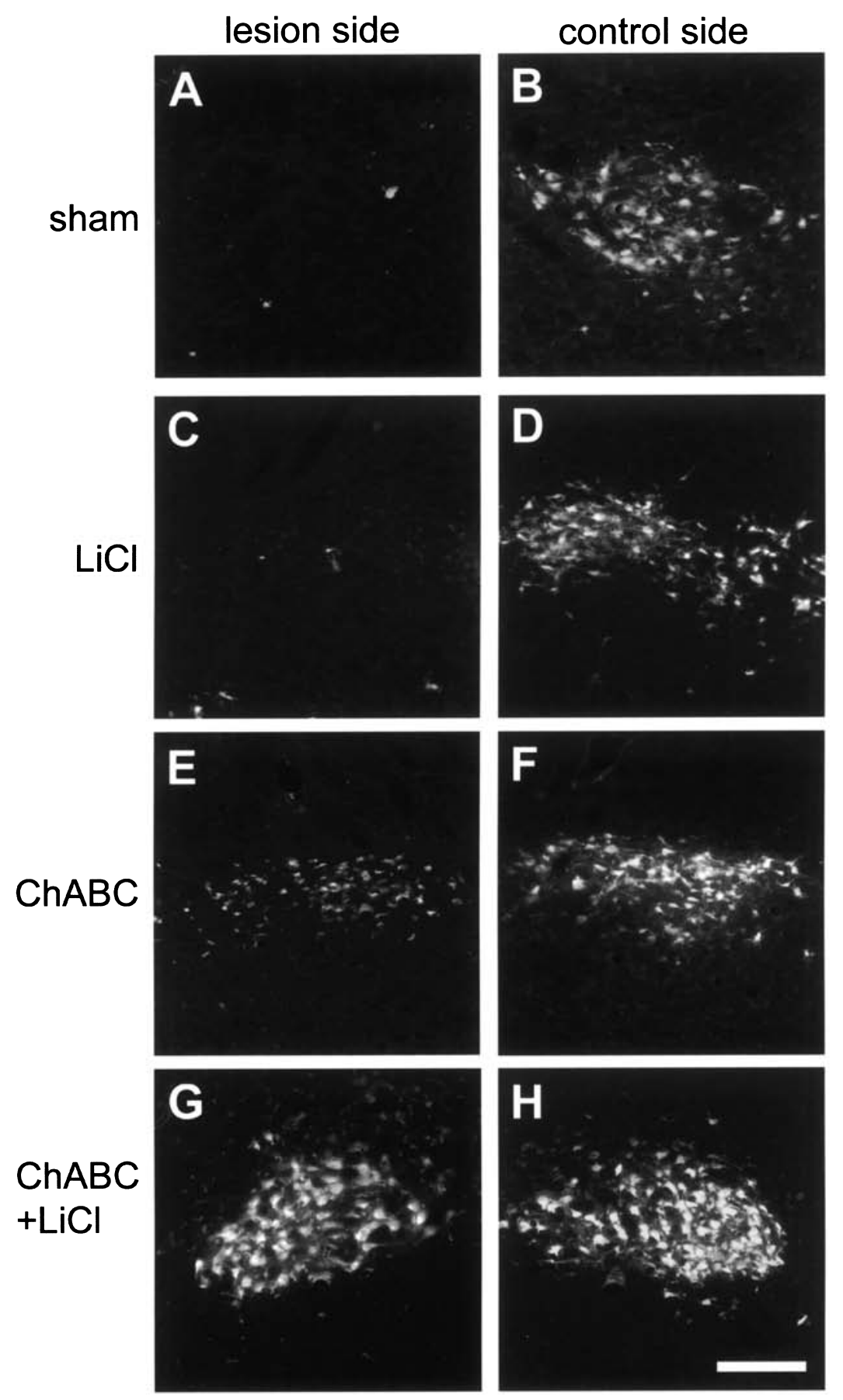

FIG. 1. Photomicrographs of midbrain cross sections showing Fluoro-Gold (FG)-labeled rubrospinal tract (RST) neurons. The animals received a right hemisection of the spinal cord at C7. The postoperative survival period was 1 month. The RST neurons in both the control side (right) and the lesion side (left) were retrogradely labeled by injection of FG at T2 spinal cord. In the sham group $(\mathbf{A}, \mathbf{B})$ and the LiCl-treated group $(\mathbf{C}, \mathbf{D})$, there are virtually no RST neurons labeled with FG on the lesion side. $(\mathbf{E}, \mathbf{F})$ In chondroitinase ABC-treated animals, few FG-labeled RST neurons are found on the lesion side. $(\mathbf{G}, \mathbf{H})$ In animals treated with $\mathrm{LiCl}$ together with chondroitinase $\mathrm{ABC}$, numerous RST neurons are labeled with FG on the left lesion side. Bar $=200 \mu \mathrm{m}$. 


\section{LITHIUM AND CHONDROITINASE ABC PROMOTE REGENERATION}

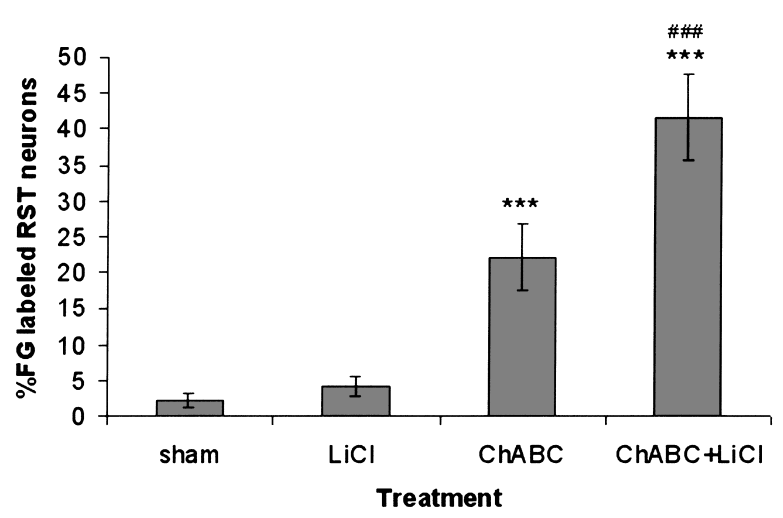

FIG. 2. Percentages of rubrospinal tract (RST) neurons labeled with Fluoro-Gold (FG) after different treatments. The animals received a right hemisection of the spinal cord at $\mathrm{C} 7$. The postoperative survival period was 1 month. In the sham and LiCl-treated animals, virtually no RST neurons are labeled by FG. Local delivery of chondroitinase ABC (ChABC) increases the percentage of FG-labeled RST neurons to $22 \%$ (sham compared with $\mathrm{ChABC} * * * p<0.001)$. In animals treated with $\mathrm{LiCl}$ together with $\mathrm{ChABC}$, there are $42 \%$ of the RST neurons labeled with FG (sham compared with $\mathrm{LiCl}+\mathrm{ChABC} * * * p<$ 0.001; ChABC compared with $\mathrm{LiCl}+\mathrm{ChABC} \# p<0.001$ ). Data are given as percentages of FG-labeled RST neurons on the lesion side against the number of FG-labeled RST neurons on the contralateral intact side, mean $\pm \mathrm{SEM}$.

amine whether $\mathrm{LiCl}$ treatment enhances the expression of Bcl-2 in RST neurons after injury of the spinal cord. Immunoblot analysis on lysates of the injured red nucleus revealed that the expression level of Bcl-2 increased 5 days after $\mathrm{LiCl}$ treatment (Fig. 4). The expression of Bcl2 was undetectable in the normal and sham control.

After spinal cord hemisection, cryosections from midbrains harvested at different time points were immunostained with an antibody against Bcl-2. In the absence of $\mathrm{LiCl}$, no $\mathrm{Bcl}-2$-expressing neurons were found on both the contralateral control and injured side of the red nucleus at all the time points examined. Treatment with $\mathrm{LiCl}$ resulted in an intense Bcl-2 immunoreactivity observed on the injured side of the red nucleus - but not on the intact control side -5 days after the operation (Fig. 6). These results indicate that Bcl-2 is involved in the additive regeneration-promoting effect of $\mathrm{LiCl}$ on axotomized RST neurons after treatment with ChABC.

\section{Behavioral Analysis}

To examine the recovery of function, a Plexiglas cylinder was used to test forelimb use during spontaneous vertical exploration 1 month after different treatments. The use of forelimbs was expressed by the percentage of wall
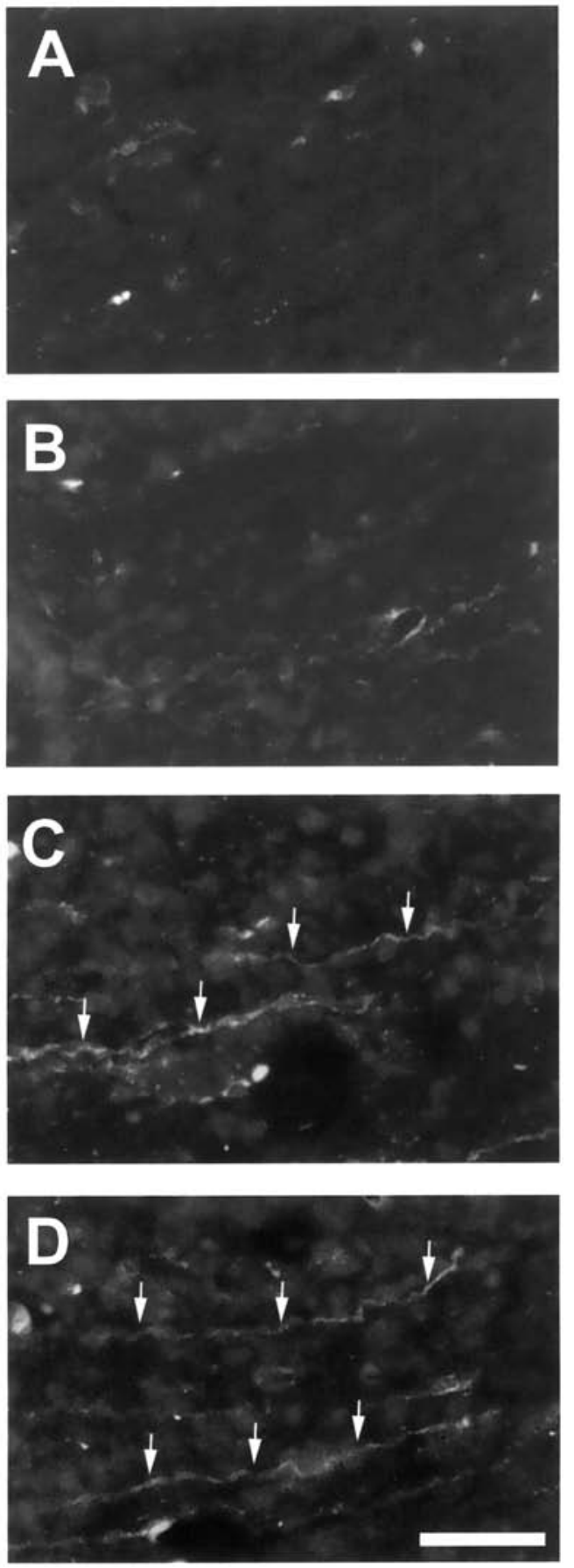

FIG. 3. Horizontal sections showing the Fluoro-Gold (FG)labeled axons within the site of spinal cord hemisection. In the sham (A) or LiCl-treated animals (B), no FG-labeled axons are seen within the lesion scar. In the animals treated with chondroitinase $\mathrm{ABC}(\mathbf{C})$ or $\mathrm{LiCl}$ together with chondroitinase $\mathrm{ABC}$ (D), a few FG-labeled axons are observed in the hemisection site being approximately 100-200 $\mu \mathrm{m}$ long (arrows) in the focal plane. Bar $=50 \mu \mathrm{m}$. 


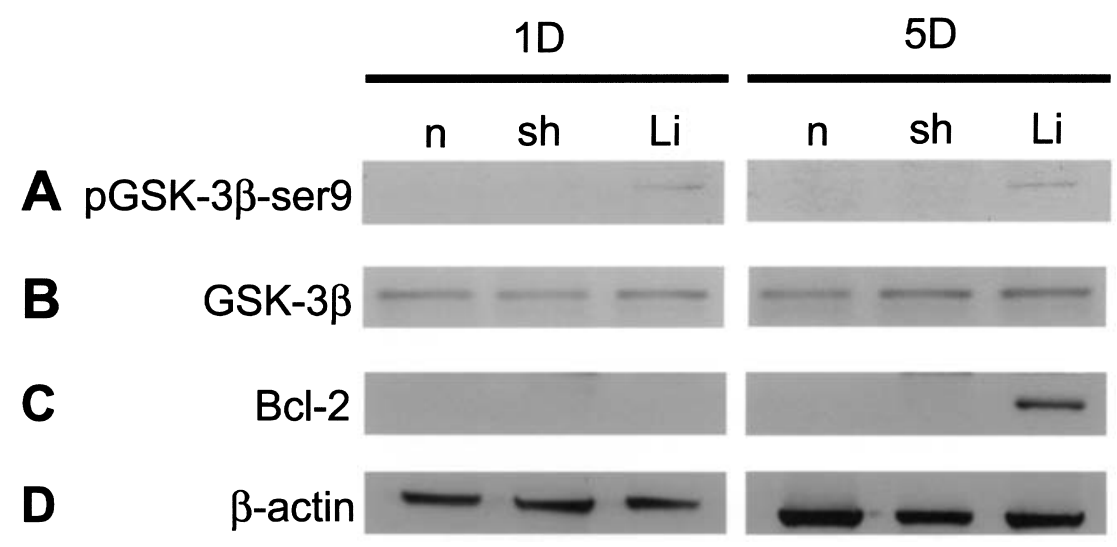

FIG. 4. Phosphorylation of GSK-3 $\beta$ and upregulation of Bcl-2 after treatment with LiCl. Fifty micrograms of total proteins from the lesion side of the red nucleus were analyzed by Western blot at 1 and 5 days after spinal cord hemisection. (A) There was no p-GSK-3 $\beta$-serine 9 immunoreactivity detected in normal (n) and sham (sh) animals. Treatment with $\mathrm{LiCl}$ increases the phosphorylation of GSK-3 $\beta$ at serine- 9 by 1 day, persisting up to 5 days, after spinal cord hemisection. (B) The expression level of phosphorylation-independent GSK-3 $\beta$ was stable throughout the experiment. (C) No Bcl-2 immunoreactivity was detected in normal and sham animals. $\mathrm{LiCl}$ treatment increased the expression of Bcl-2 by 5 days after spinal cord hemisection. (D) $\beta$-actin staining was used as an internal control.

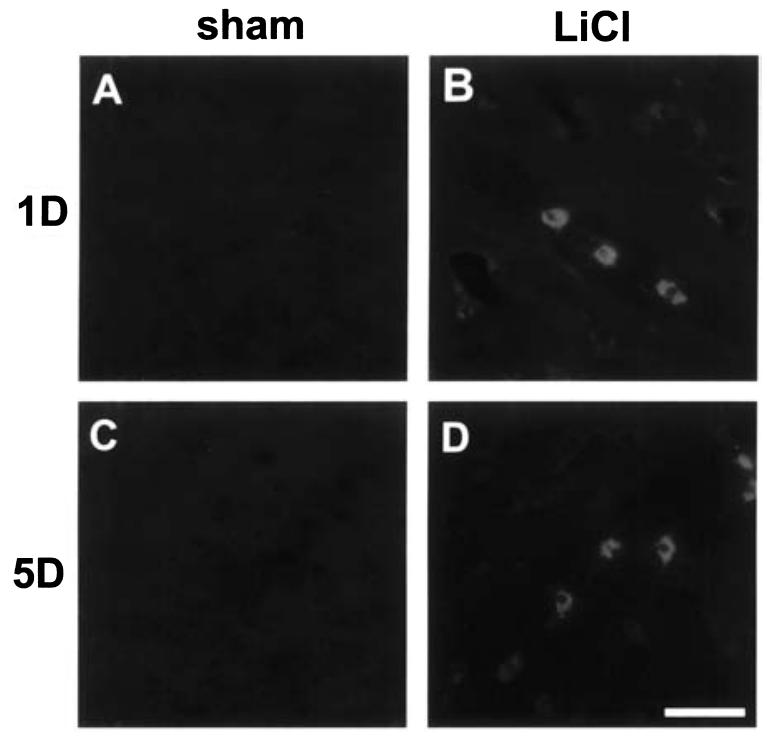

FIG. 5. Photomicrographs of midbrain cross sections showing the expression of the phosphorylated serine- 9 of GSK-3 $\beta$ in rubrospinal tract neurons on the lesion side. After hemisection of the $\mathrm{C} 7$ spinal cord, the animals received daily intraperitoneal injections of $\mathrm{LiCl}$. (A,C) In the sham group, no p-GSK-3 $\beta$-serine-9 immunoreactive rubrospinal tract neurons are found by 1 and 5 days after the operation. (B,D) In LiCltreated animals, neurons showing positive immunoreactivity for p-GSK- $3 \beta$-serine- 9 are found in the RST nucleus by 1 and 5 days. $\mathrm{Bar}=50 \mu \mathrm{m}$.
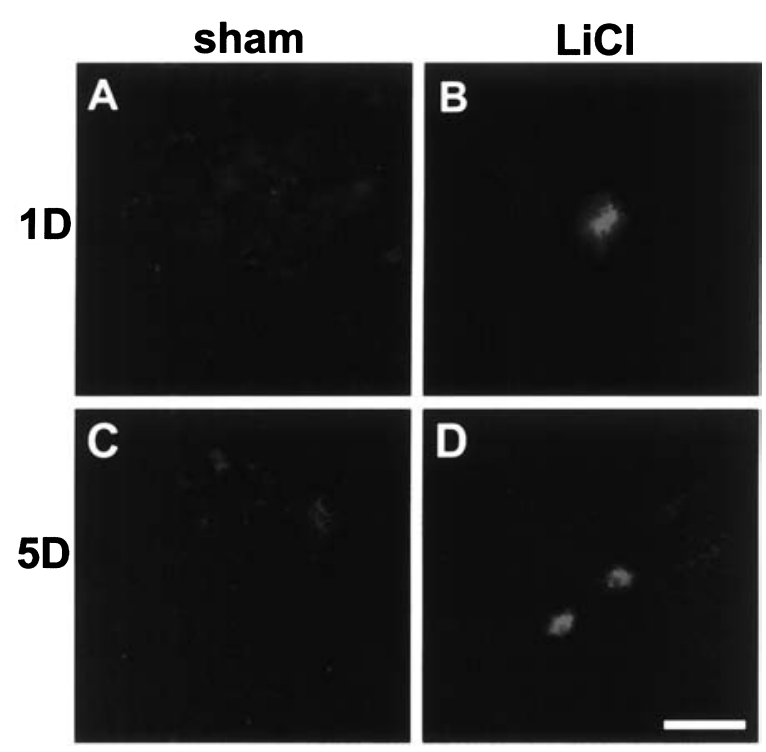

FIG. 6. Photomicrographs of midbrain cross sections showing the expression of the $\mathrm{Bcl}-2$ in rubrospinal tract neurons on the lesion side. After hemisection of the $\mathrm{C} 7$ spinal cord, the animals received daily intraperitoneal injections of $\mathrm{LiCl}$. (A,C) In the sham group, no Bcl-2 immunoreactive RST neurons are found by 1 and 5 days after the operation. (B,D) In LiCl-treated animals, neurons showing positive immunoreactivity for Bcl2 are found in the RST nucleus by 1 and 5 days. Bar $=50 \mu \mathrm{m}$. 


\section{LITHIUM AND CHONDROITINASE ABC PROMOTE REGENERATION}

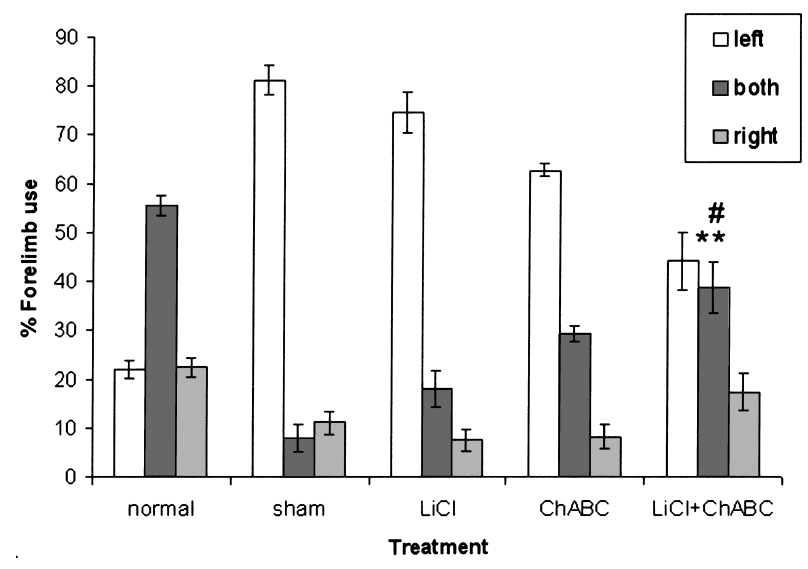

FIG. 7. Behavioral analysis of forelimb function in vertical exploration test. Normal animals use a single forelimb or both forelimbs together for vertical exploration. One month after spinal cord hemisection, animals in the sham and LiCl-treated groups rarely use their right (impaired) forelimb. Chondroitinase $\mathrm{ABC}(\mathrm{ChABC})$-treated animals use both forelimbs more frequently for exploration. Animals treated with $\mathrm{LiCl}$ together with ChABC showed significant use of both forelimbs compared to the sham and single treated animals. Data are mean \pm SEM. Sham compared with ChABC **p $<0.01$. ChABC com-

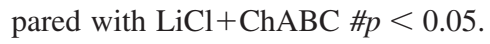

exploration that was initiated by right (impaired) or left (normal) forelimb alone or by both forelimbs together (Fig. 7). Normal rats explored the cylinder wall using either a single forelimb (about 45\%) or both forelimbs together (about 55\%). Animals receiving hemisection at C7 rarely used the forelimb ipsilateral to the injury (sham group, $7.7 \pm 3.0 \%$ ), leading to asymmetry of forelimb use. Animals treated with $\mathrm{LiCl}$ used both forelimbs slightly more often, but the difference was not significant from the sham group $(18.1 \pm 3.7 \%$; sham compared with $\mathrm{LiCl}, p>0.05)$. In the ChABC-treated group, rats used both forelimbs more frequently than did the sham group (29.1 $\pm 1.9 \%$; sham compared with ChABC, $p<0.01)$, resulting in more symmetrical forelimb use. However, combined treatment of $\mathrm{LiCl}$ together with $\mathrm{ChABC}$ significantly promoted the use of both forelimbs as compared with all other treatment groups $(38.7 \pm 5.2 \%$; sham compared with $\mathrm{LiCl}+\mathrm{ChABC}, p<0.01 ; \mathrm{LiCl}$ compared with $\mathrm{LiCl}+\mathrm{ChABC}, p<0.01$; ChABC compared with $\mathrm{LiCl}+\mathrm{ChABC}, p<0.05)$. Rats receiving the combined treatment used both forelimbs together $(38.7 \pm 5.2 \%)$ as frequently as they used their unimpaired forelimb alone $(44.1 \pm 5.9 \%$; both forelimbs compared with unaffected limb, $p>0.05)$. In addition, the animals receiving the combined treatment used their impaired forelimb alone to explore the wall more frequently compared with all the other treatment groups $(17.3 \pm 3.8 \%$; sham compared with $\mathrm{LiCl}+\mathrm{ChABC}, p<0.01 ; \mathrm{LiCl}$ compared with $\mathrm{LiCl}+\mathrm{ChABC}, \quad p<0.01 ; \mathrm{ChABC}$ compared with $\mathrm{LiCl}+\mathrm{ChABC}, p<0.05)$. These results demonstrate that treatment with $\mathrm{ChABC}$, but not $\mathrm{LiCl}$ alone, partially promotes the recovery of function after spinal cord injury. Most interestingly, combined treatment of $\mathrm{LiCl}$ together with ChABC significantly increases the extent of recovery as compared with $\mathrm{ChABC}$ treatment alone.

\section{DISCUSSION}

Enzymatic degradation of CSPGs promotes the axonal regeneration of neurons across the lesion scar following CNS injuries. Lithium has been shown to protect neurons and enhance axonal regrowth potential via GSK-3 $\beta$ and Bcl-2 signaling pathways (Bournat et al., 2000; Huang et al., 2003). In this study, we provide in vivo evidence that application of ChABC at the lesion site promotes the axonal regeneration of RST neurons after injury of the spinal cord. Most important, combined treatment of $\mathrm{LiCl}$ together with ChABC further enhances the regeneration of RST neurons following this injury. This combined treatment can improve the forelimb function in a vertical exploration test. Western blot and immunohistochemical analyses reveal that $\mathrm{LiCl}$ treatment induces the expression of the p-GSK-3 $\beta$-serine- 9 as well as the upregulation of Bcl-2 in severed RST neurons. These results indicate that in axotomized RST neurons, lithium reinforces the regeneration-promoting effect of $\mathrm{ChABC}$ via a mechanism involving the inactivation of GSK-3 $\beta$ and upregulation of Bcl-2. Our study confirms previously published data indicating that enzymatic removal of CSPGs promotes axonal regrowth of injured neurons and that Bcl2 is involved in the neuroprotective effect of lithium.

Previous strategies for promoting the axonal regeneration of injured RST have been focused on the use of neurotrophic factors. In a model of chronic spinal cord injury, continuous administration of BDNF at the midbrain reverses massive atrophy and promotes regeneration of rubrospinal axons into a peripheral nerve implanted at the lesion site (Kwon et al., 2002). Following hemisection of the spinal cord, transplants of fibroblasts genetically modified to express BDNF prevent atrophy, enhance axonal regeneration of rubrospinal neurons, and result in partial recovery of forelimb function (Liu et al., 1999, 2002; Jin et al., 2002). It has been suggested that neurotrophic factors promote the regeneration of axotomized rubrospinal neurons by stimulating the expression of GAP-43 and T $\alpha 1$-tubulin (Kobayashi et al., 1997; Fernandes et al., 1999). 


\section{YICK ET AL.}

We have previously shown that enzymatic degradation of CSPGs at the injury scar can enhance the regeneration of the ascending Clarke's neurons after spinal cord hemisection (Yick et al., 2000). Removing the glycosaminoglycan chains of CSPGs also promotes the regeneration of severed axons in nigrostriatal and corticospinal tracts (Moon et al., 2001; Bradbury et al., 2002). In this study, we demonstrate that CSPGs at the injury scar are important inhibitory factors for axon regeneration of severed rubrospinal neurons. Taken together, these in vivo studies provide evidence that CSPGs are critical inhibitory molecules against axonal regeneration in the injured CNS. We are investigating whether multiple treatment with $\mathrm{ChABC}$ would be more effective in promoting axonal regeneration after spinal cord injury.

Although its precise mechanism of action remains unknown, lithium is a commonly used drug for the treatment of mania and depression (Post et al., 1992). An in vitro study has demonstrated that lithium supports the cell survival and axonal regeneration of retinal ganglion neurons (Huang et al., 2003). Long-term exposure to lithium significantly protects cultured cerebellar, cerebral cortical, and hippocampal neurons against glutamate-induced apoptotic neuronal death (Nonaka et al., 1998). In a rat model of Huntington's disease, subcutaneous administration of lithium reduces the size of quinolinic acid-induced lesion and protects striatal neurons from apoptotic death (Wei et al., 2001). Long-term lithium treatment also protects cerebellar granule cell neurons from $\beta$-amyloid peptide-induced death (Wei et al., 2000). Lithium can prevent potassium efflux-induced neuronal cell apoptosis, with the induction of caspase- 3 expression ( $\mathrm{Li}$ and El-Mallahk, 2000). Our results show that treatment of $\mathrm{LiCl}$ alone fails to promote the regeneration of severed $\mathrm{RST}$ axons. Interestingly, $\mathrm{LiCl}$ seems to potentiate the neuroregenerative function of $\mathrm{ChABC}$, resulting in an additive effect that promotes axonal regeneration of injured RST neurons. One of the possibilities is that $\mathrm{LiCl}$ protects the injured RST neurons from death so that more axons can regenerate across the growth-permissive lesion scar after ChABC treatment. It is also possible that lithium binds to CSPGs and hyaluronic acid and changes the water-binding capacity of GAGs, resulting in a synergistic effect of $\mathrm{LiCl}$ and $\mathrm{Ch} \mathrm{ABC}$ on axonal regeneration.

We have found that the number of surviving neurons decreases in the injured red nucleus both in the sham controls and following lithium treatment (data not shown). This result is different from a previous study, which showed that axotomized RST neurons survive for 1 year with regrowth by applying brain-derived neurotrophic factor (Kwon et al., 2002). It is possible that the dose of $\mathrm{LiCl}$ we used can increase the expression of $\mathrm{Bcl}-2$ but is not optimal for promoting the survival of injured RST neurons. We cannot rule out the possibility, too, that the number of surviving neurons is underestimated because only large and morphologically normal neurons are counted, and not the cells with massive atrophy. Further studies are required to determine whether $\mathrm{LiCl}$ can promote the survival of axotomized RST neurons after spinal cord injury.

Here, we demonstrate that the number of FG-labeled RST neurons is significantly increased after the combined treatment of $\mathrm{LiCl}$ together with $\mathrm{ChABC}$. The number of RST neurons that are labeled for $\mathrm{p}-\mathrm{GSK}-3 \beta$-serine 9 or for Bcl-2 is limited. This result indicates that in addition to inactivation of GSK-3 $\beta$ and upregulation of $\mathrm{Bcl}-2$, other mechanisms may be involved in the synergistic effect of $\mathrm{LiCl}$ and $\mathrm{ChABC}$.

There is growing evidence that GSK- $3 \beta$ signaling is involved in neurodegeneration by modulating neuronal apoptosis. GSK- $3 \beta$ is activated by phosphorylation at Tyr216 kinase domain and is inactivated through phosphorylation of serine-9 (Kaylor and Orr, 2002). The executive phase of apoptosis is associated with a selective phosphorylation of serine-9 together with dephosphorylation of tyrosine-216 in GSK-3 $\beta$ (Bhat et al., 2000; Elyaman et al., 2002). Lithium is a selective inhibitor of GSK$3 \beta$, which may in turn regulate transcriptional factors such as nuclear factor kappa-B (Bournat et al., 2000) and cyclic AMP response element binding protein (Grimes and Jope, 2001). The activity of GSK-3 $\beta$ is negatively regulated by signal transduction cascades that protect neurons against apoptosis, including the PI-3 kinase-Akt pathways (Hetman et al., 2000). By using a neuronal apoptosis model in neonatal rats, it has been demonstrated that $\mathrm{LiCl}$ can prevent GSK-3 $\beta$-mediated Tau phosphorylation in response to a NMDA receptor antagonist (Elyaman et al., 2002). Our Western blot and immunohistochemical analyses provide in vivo evidence that after spinal cord hemisection, $\mathrm{LiCl}$ treatment inhibits GSK-3 $\beta$ by phosphorylation at serine- 9 in the red nucleus.

In addition to GSK- $3 \beta$ inhibition, it has been recently demonstrated that lithium regulates the expression of antiapoptotic proteins like Bcl-2. Bcl-2 is an antiapoptotic gene that inhibits Bax-mediated release of cytochrome $c$ or apoptosis-inducing factors from mitochondria, thereby preventing caspase activation of cell death. In a culture model, lithium increases the expression of Bcl-2 mRNA, as well as promotes both the survival and axonal regeneration of retinal ganglion cells (Huang et al., 2003). In a rat model of Huntington's disease, lithium treatment increases Bcl-2 protein levels and suppresses excitotoxininduced striatal lesions (Wei et al., 2001). Under glutamate excitotoxicity, long-term treatment of cultured cerebellar granule cells with lithium upregulates the 


\section{LITHIUM AND CHONDROITINASE ABC PROMOTE REGENERATION}

mRNA and protein levels of Bcl-2 remarkably (Chen and Chuang, 1999). In this study, immunoblotting and immunohistochemical analyses reveal that Bcl-2 is upregulated in the injured red nucleus after $\mathrm{LiCl}$ treatment. It is possible that $\mathrm{LiCl}$ reinforces the neuroregenerative-promoting effect of $\mathrm{ChABC}$ by mediating the upregulation of $\mathrm{Bcl}-2$ expression in injured RST neurons. However, further studies are required to examine the temporal relationship between Bcl-2 upregulation and RST axonal regeneration after spinal cord injury.

In our study, administration of $\mathrm{LiCl}$ alone did not promote RST regeneration, but it enhanced the regenerationpromoting function of ChABC on injured RST. It has been demonstrated that the role of lithium on enhancing neuronal survival may involve inhibition of NMDA receptors, downregulation of proapoptotic genes such as p53 and Bax, increased expression of the neuroprotective protein $\mathrm{Bcl}-2$, and activation of the cell survival kinase Akt. However, the role of $\mathrm{LiCl}$ in the promotion of axonal regeneration is unclear. Bcl-2 plays a key role in promoting regeneration of injured axons in the CNS (Chen et al., 1997). Lithium enhances the axonal regeneration of retinal ganglion cells through a Bcl-2-dependent mechanism (Huang et al., 2003). Further studies are required to understand the precise mechanism of lithiumpromoted axonal regeneration in the injured CNS.

On the basis of observations of vertical exploring activity with forelimbs in a cylinder, our behavioral result shows that treatment with $\mathrm{ChABC}$ reduces the asymmetry of forelimb use after injury of the spinal cord. Interestingly, combined treatment of $\mathrm{LiCl}$ together with ChABC further relieves the asymmetry of forelimb use compared with ChABC treatment alone. Treatment with $\mathrm{LiCl}$ alone does not improve forelimb function. These results are consistent with our histological data, showing that the number of regenerating RST neurons is increased in the animals with the combined treatment compared with $\mathrm{ChABC}$ application alone.

This study provides in vivo evidence that application of ChABC promotes the axonal regeneration of RST neurons after spinal cord injury. Most interestingly, combined treatment of $\mathrm{ChABC}$ together with $\mathrm{LiCl}$ results in an additive effect on promoting RST axonal regeneration. The combined treatment enhances the recovery of forelimb function compared with a single application of $\mathrm{ChABC}$ alone. Treatment with $\mathrm{LiCl}$ inhibits the activity of GSK- $3 \beta$ and upregulates the expression of Bcl-2 in axotomized RST neurons. These results indicate that $\mathrm{LiCl}$ reinforces the neuroregeneration-promoting function of ChABC via inhibiting GSK- $3 \beta$ and upregulating Bcl-2. To improve the regenerative response after CNS injury, it will be important to develop intervention that alters the CSPG components of the glial scar as well as stimulates the intrinsic regrowth capability of the injured neurons. Combined use of lithium together with ChABC may be a novel strategy for the treatment of spinal cord injury.

\section{ACKNOWLEDGMENTS}

We are grateful to Dr. Peter Lucas of the Department of Anatomy at The University of Hong Kong for his helpful comments on the manuscript. This study was supported by research grants from The University of Hong Kong and The National Key Basic Research Programme of China (2003CB515303).

\section{REFERENCES}

BHAT, R.V., SHANLEY, J., CORRELL, M.P., et al. (2000). Regulation and localization of tyrosine 216 phosphorylation of glycogen synthase kinase- $3_{\text {beta }}$ in cellular and animal models of neuronal degeneration. Proc. Natl. Acad. Sci. USA. 97, 11074-11079.

BOURNAT, J.C., BROWN, A.M., and SOLER, A.P. (2000). Wnt-1-dependent activation of the survival factor NF-kappaB in PC12 cells. J. Neurosci. Res. 61, 21-32.

BOVOLENTA, P., FERNAUD-ESPINOSA, I., MENDEZOTERO, R., et al. (1997). Neurite outgrowth inhibitor of gliotic brain tissue. Mode of action and cellular localization, studied with specific monoclonal antibodies. Eur. J. Neurosci. 9, 977-989.

BRADBURY, E.J., MOON, L.D., POPAT, R.J., et al. (2002). Chondroitinase $\mathrm{ABC}$ promotes functional recovery after spinal cord injury. Nature 416, 636-640.

BROWN, L.T. (1974). Rubrospinal projections in the rat. J. Comp. Neurol. 154, 169-187.

CARONI, P., and SCHWAB, M.E. (1988). Antibody against myelin-associated inhibitor of neurite growth neutralizes nonpermissive substrate properties of CNS white matter. Neuron 1, 85-96.

CHEN, D.F., SCHNEIDER, G.E., MARTINOU, J.C., et al. (1997) Bcl-2 promotes regeneration of severed axons in mammalian CNS. Nature 385, 434-439.

CHEN, G., HUANG, L.D., JIANG, Y.M., et al. (1999). The mood-stabilizing agent valproate inhibits the activity of glycogen synthase kinase-3. J. Neurochem. 72, 1327-1330.

CHEN, R.W., and CHUANG, D.M. (1999). Long term lithium treatment suppresses p53 and Bax expression but increases Bcl-2 expression. A prominent role in neuroprotection against excitotoxicity. J. Biol. Chem. 274, 6039-6042.

CHIERZI, S., STRETTOI, E., CENNI, M.C., et al. (1999). Optic nerve crush: axonal responses in wild-type and bcl-2 transgenic mice. J. Neurosci. 19, 8367-8376. 


\section{YICK ET AL.}

CROWDER, R.J., and FREEMAN, R.S. (2000). Glycogen synthase kinase-3 beta activity is critical for neuronal death caused by inhibiting phosphatidylinositol 3-kinase or Akt but not for death caused by nerve growth factor withdrawal. J. Biol. Chem. 275, 34266-34271.

DIENER, P.S., and BREGMAN, B.S. (1998). Fetal spinal cord transplants support growth of supraspinal and segmental projections after cervical spinal cord hemisection in the neonatal rat. J. Neurosci. 18, 779-793.

DOU, C.L., and LEVINE, J.M. (1994). Inhibition of neurite growth by the NG2 chondroitin sulfate proteoglycan. J. Neurosci. 14, 7616-7628.

ELYAMAN, W., TERRO, F., WONG, N.S., et al. (2002). In vivo activation and nuclear translocation of phosphorylated glycogen synthase kinase- $3_{\text {beta }}$ in neuronal apoptosis: links to tau phosphorylation. Eur. J. Neurosci. 15, 651-660.

FERNANDES, K.J., FAN, D.P., TSUI, B.J., et al. (1999). Influence of the axotomy to cell body distance in rat rubrospinal and spinal motoneurons: differential regulation of GAP-43, tubulins, and neurofilament-M. J. Comp. Neurol. 414, 495-510.

FIDLER, P.S., SCHUETTE, K., ASHER, R.A., et al. (1999). Comparing astrocytic cell lines that are inhibitory or permissive for axon growth: the major axon-inhibitory proteoglycan is NG2. J. Neurosci. 19, 8778-8788.

GRIMES, C.A., and JOPE, R.S. (2001). CREB DNA binding activity is inhibited by glycogen synthase kinase- 3 beta and facilitated by lithium. J. Neurochem. 78, 1219-1232.

HETMAN, M., CAVANAUGH, J.E., KIMELMAN, D., et al. (2000). Role of glycogen synthase kinase-3beta in neuronal apoptosis induced by trophic withdrawal. J. Neurosci. 20, 2567-2574.

HIMES, B.T., GOLDBERGER, M.E., and TESSLER, A. (1994). Grafts of fetal central nervous system tissue rescue axotomized Clarke's nucleus neurons in adult and neonatal operates. J. Comp. Neurol. 339, 117-131.

HOLM, K., and ISACSON, O. (1999). Factors intrinsic to the neuron can induce and maintain its ability to promote axonal outgrowth: a role for BCL2? Trends Neurosci. 22, 269-273.

HUANG, X., WU, D.Y., CHEN, G., et al. (2003). Support of retinal ganglion cell survival and axon regeneration by lithium through a Bcl-2-dependent mechanism. Invest. Ophthalmol. Vis. Sci. 44, 347-354.

JIN, Y., FISCHER, I., TESSLER, A., et al. (2002). Transplants of fibroblasts genetically modified to express BDNF promote axonal regeneration from supraspinal neurons following chronic spinal cord injury. Exp. Neurol. 177, 265-275.

KAYTOR, M.D., and ORR, H.T. (2002). The GSK3 beta signaling cascade and neurodegenerative disease. Curr. Opin. Neurobiol. 12, 275-278.

KOBAYASHI, N.R., FAN, D.P., GIEHL, K.M., et al. (1997). BDNF and NT-4/5 prevent atrophy of rat rubrospinal neu- rons after cervical axotomy, stimulate GAP-43 and Talpha1tubulin mRNA expression, and promote axonal regeneration. J. Neurosci. 17, 9583-9595.

KWON, B.K., LIU, J., MESSERER, C., et al. (2002). Survival and regeneration of rubrospinal neurons 1 year after spinal cord injury. Proc. Natl. Acad. Sci. USA. 99, 3246-3251.

LI, R., and EL-MALLAHK, R.S. (2000). A novel evidence of different mechanisms of lithium and valproate neuroprotective action on human SY5Y neuroblastom cells: caspase-3 dependency. Neurosci. Lett. 294, 147-150.

LIESI, P., and KAUPPILA, T. (2002). Induction of type IV collagen and other basement-membrane-associated proteins after spinal cord injury of the adult rat may participate in formation of the glial scar. Exp. Neurol. 173, 31-45.

LIU, Y., KIM, D., HIMES, B.T., et al. (1999). Transplants of fibroblasts genetically modified to express BDNF promote regeneration of adult rat rubrospinal axons and recovery of forelimb function. J. Neurosci. 19, 4370-4387.

LIU, Y., HIMES, B.T., MURRAY, M., et al. (2002). Grafts of BDNF-producing fibroblasts rescue axotomized rubrospinal neurons and prevent their atrophy. Exp. Neurol. 178, $150-164$.

MANJI, H.K., MOORE, G.J., RAJKOWSKA, G., et al. (2000). Neuroplasticity and cellular resilience in mood disorders. Mol. Psychiatry 5, 578-593.

MASON, M.R., LIEBERMAN, A.R., GRENNINGLOH, G., et al. (2002). Transcriptional upregulation of SCG10 and CAP-23 is correlated with regeneration of the axons of peripheral and central neurons in vivo. Mol. Cell. Neurosci. 20, 595-615.

MOON, L.D.F., ASHER, R.A., RHODES, K.E., et al. (2001). Regeneration of CNS axons back to their target following treatment of adult rat brain with chondroitinase ABC. Nat. Neurosci. 4, 465-466.

NONAKA, S., HOUGH, C.J., and CHUANG, D.M. (1998). Chronic lithium treatment robustly protects neurons in the central nervous system against excitotoxicity by inhibiting $\mathrm{N}$-methyl-D-aspartate receptor-mediated calcium influx. Proc. Natl. Acad. Sci. USA 95, 2642-2647.

PAP, M., and COOPER, G.M. (1998). Role of glycogen synthase kinase-3 in the phosphatidylinositol 3-Kinase/Akt cell survival pathway. J. Biol. Chem. 273, 19929-19932.

POST, R.M., WEISS, S.R., and CHUANG, D.M. (1992). Mechanisms of action of anticonvulsants in affective disorders: comparisons with lithium. J. Clin. Psychopharmacol. 12, 23S-35S.

RICHARDSON, P.M., McGUINNESS, U.M., and AGUAYO, A.J. (1982). Peripheral nerve autografts to the rat spinal cord: studies with axonal tracing methods. Brain Res. 237, 147-162.

SCHNELL, L., and SCHWAB, M.E. (1990). Axonal regeneration in the rat spinal cord produced by an antibody against 


\section{LITHIUM AND CHONDROITINASE ABC PROMOTE REGENERATION}

myelin-associated neurite growth inhibitors. Nature 343, 269-272.

SCHWAB, J.M., SEID, K., and SCHLUESENER, H.J. (2001). Traumatic brain injury induces prolonged accumulation of cyclooxygenase-1 expressing microglia/brain macrophages in rats. J. Neurotrauma 18, 881-890.

SHIBAYAMA, M., HATTORI, S., HIMES, B.T., et al. (1998). Neurotrophin-3 prevents death of axotomized Clarke's nucleus neurons in adult rat. J. Comp. Neurol. 390, 102-111.

THALLMAIR, M., METZ, G.A., Z'GRAGGEN, W.J., et al. (1998). Neurite growth inhibitors restrict plasticity and functional recovery following corticospinal tract lesions. Nat. Neurosci. 1, 124-131.

WANG, X., MESSING, A., and DAVID, S. (1997). Axonal and nonneuronal cell responses to spinal cord injury in mice lacking glial fibrillary acidic protein. Exp. Neurol. 148, $568-576$.

WEI, H., LEEDS, P.R., QIAN, Y., et al. (2000). beta-amyloid peptide-induced death of PC 12 cells and cerebellar granule cell neurons is inhibited by long-term lithium treatment. Eur. J. Pharmacol. 392, 117-123.

WEI, H., QIN, Z.H., SENATOROV, V.V., et al. (2001). Lithium suppresses excitotoxicity-induced striatal lesions in a rat model of Huntington's disease. Neuroscience 106, 603-612.
XU, X.M., and MARTIN, G.F. (1991). Evidence for new growth and regeneration of cut axons in developmental plasticity of the rubrospinal tract in the North American opossum. J. Comp. Neurol. 313, 103-112.

YICK, L.W., CHEUNG, P.T., SO, K.F., et al. (2003). Axonal regeneration of Clarke's neurons beyond the spinal cord injury scar after treatment with chondroitinase ABC. Exp. Neurol. 182, 160-168.

YICK, L.W., WU, W., SO, K.F., et al. (2000). Chondroitinase ABC promotes axonal regeneration of Clarke's neurons after spinal cord injury. NeuroReport. 11, 1063-1067.

YUKAWA, Y., LOU, J., FUKUI, N., et al. (2002). Optimal treatment timing to attenuate neuronal apoptosis via $\mathrm{Bcl}-2$ gene transfer in vitro and in vivo. J. Neurotrauma 19, 1091-103.

Address reprint requests to: Wu-Tian Wu, M.D. Department of Anatomy The University of Hong Kong Faculty of Medicine Building

21 Sassoon Road Hong Kong

E-mail: wtwu@hkucc.hku.hk 